Supporting Information for:

\title{
Shrinking the Synchrotron: Tabletop Extreme Ultraviolet Absorption of Transition Metal Complexes
}

Kaili Zhang, Ming-Fu Lin, Elizabeth S. Ryland, Max A. Verkamp, Kristopher Benke, Frank M. F. de Groot, Gregory S. Girolami, Josh Vura-Weis

\section{Oxidation state specificity}

Two additional samples have been measured to demonstrate the robustness of the oxidation state specificity. Figure $\mathrm{S} 1$ below reproduces the spectra of $\mathrm{Co}^{\mathrm{II}}(\mathrm{Tp})_{2}, \mathrm{Co}^{\mathrm{II}} \mathrm{OEP}$, and $\mathrm{Co}^{\mathrm{III}}(\mathrm{acac})_{3}$ from the main text, with the addition of $(\mathrm{TBA})_{2} \mathrm{Co}^{\mathrm{II}} \mathrm{Cl}_{4}(\mathrm{TBA}=$ tetrabutylammonium) and $\mathrm{Co}^{\mathrm{III}}(\mathrm{Tp})_{2} \mathrm{Br}$. The $\mathrm{Co}^{\mathrm{II}}$ complexes all show a rising edge at $\sim 57.5 \mathrm{eV}$, while the $\mathrm{Co}{ }^{\mathrm{III}}$ complexes begin to rise at $\sim 62.5 \mathrm{eV}$. The $0.4 \mathrm{eV}$ shift between the rising edges of $\mathrm{Co}^{\mathrm{III}}(\mathrm{acac})_{3}$ and $\mathrm{Co}^{\mathrm{III}}(\mathrm{Tp})_{2} \mathrm{Br}$ (measured at half height) is much smaller than the $\mathrm{Co}^{\mathrm{II}}-\mathrm{Co}^{\mathrm{III}}$ shift, and likely reflects small changes in the effective nuclear charge and ligand field symmetry between the two complexes. A future publication will explore the effects of such changes on the spectral position and shape. We note that we were unable to prepare spincast films of $\mathrm{Co}^{\mathrm{III}}(\mathrm{Tp})_{2} \mathrm{Br}$ and (TBA $)_{2} \mathrm{Co}^{\mathrm{II}} \mathrm{Cl}_{4}$ that were as smooth as those of the two iron complexes, resulting in spectra with significant noise before the absorption edge (see SI, section 6). ${ }^{4}$ The fine details of the spectra (such as the relative height of the $\sim 59 \mathrm{eV}$ peak for $(\mathrm{TBA})_{2} \mathrm{Co}^{\mathrm{II}} \mathrm{Cl}_{4} \mathrm{Vs} \mathrm{Co}{ }^{\mathrm{II}}(\mathrm{Tp})_{2}$ should therefore be treated with caution. Finally, $\mathrm{Br} 3 \mathrm{~d}$ photoionization occurs at $\sim 70 \mathrm{eV}$, so the post-edge features of $\mathrm{Co}^{\mathrm{III}}(\mathrm{Tp}){ }_{2} \mathrm{Br}$ may have some contribution from $\mathrm{Br}$ absorption (likely the cause of the rising feature at $72 \mathrm{eV}$ ).

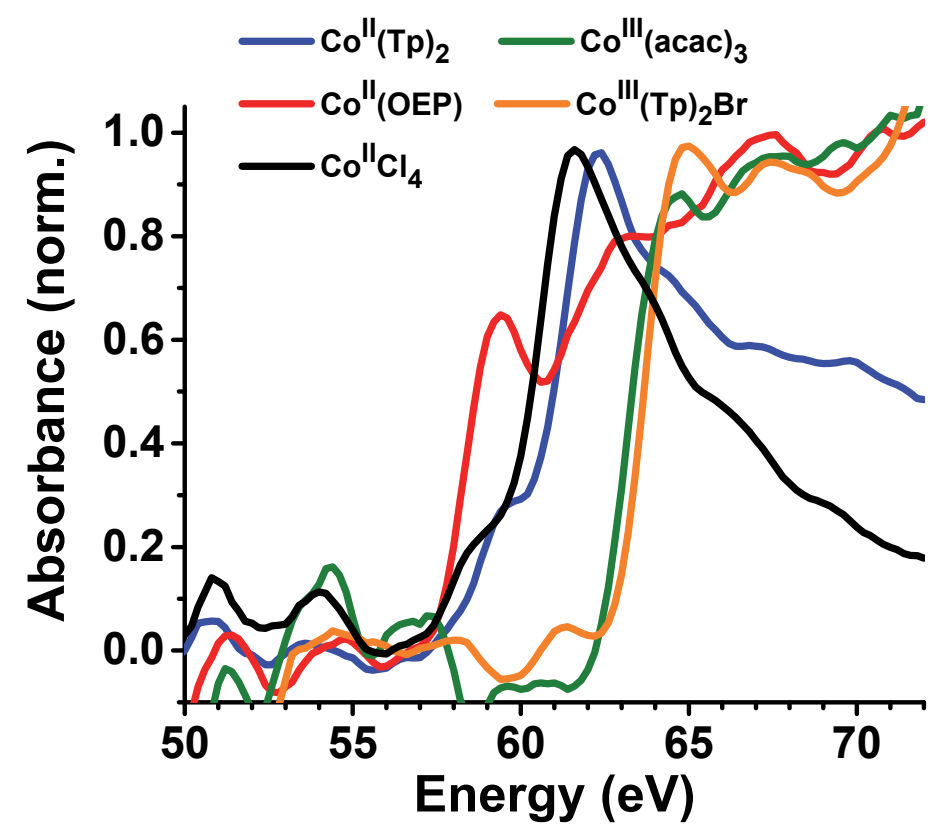

Figure S1: Spectra of CoII and CoIII complexes 


\section{Ligand Field Multiplet calculations}

Simulated spectra are computed using programs developped by Cowan ${ }^{1}$ and co-workers and modified by Thole ${ }^{2}$. The LFM model treats all electron-electron interactions using the HartreeFock approximation and all metal-ligand interactions using an ionic crystal field model. SlaterCondon factors are downscaled from the Hartree-Fock-derived values to recover the effect of electron-electron correlation not treated in Hartree-Fock and to model the nephelauxetic effect. ${ }^{1}$ The Hartree-Fock $d-d$ and $p-d$ integrals are multiplied by $0.5 .{ }^{3,4}$ Matrix elements of the Hamiltonian operator and the transition dipole operator are transplanted from the free ion into the lower symmetry of the ligand field using the Wigner-Eckart theorem. ${ }^{5}$ A simulated $\mathrm{M}_{2,3}$-edge stick spectrum for a $3 d^{\mathrm{N}}$ metal center is produced by combining all electric dipole transitions from the ground state to all terms under the $3 p^{5} 3 d^{\mathrm{N}+1}$ configuration. The crystal field splitting parameter $10 \mathrm{Dq}$ for each complex was taken from $\mathrm{UV} / \mathrm{Visible}$ or $\mathrm{L}_{2,3}$-edge studies. Values of $1.24 \mathrm{eV}, 2.89 \mathrm{eV}, 1.9 \mathrm{eV}$, and $0.7 \mathrm{eV}$ were used for $\mathrm{Co}^{\mathrm{II}}(\mathrm{Tp})_{2},{ }^{6} \mathrm{Co}^{\mathrm{III}}(\mathrm{acac})_{3}{ }^{7}$ $\mathrm{Fe}^{\mathrm{II}} \mathrm{Tren}(\mathrm{Py}){ }_{3}{ }^{2+}$, and $\mathrm{Fe}^{\mathrm{II}} \mathrm{Tren}(6-\mathrm{MePy}){ }_{3}{ }^{2+},{ }^{2}$ respectively. For square-planar $\mathrm{Co}^{\mathrm{II}} \mathrm{OEP}$, the crystal field parameters used were $10 \mathrm{Dq}=3.49 \mathrm{eV}$, Ds $=0.39 \mathrm{eV}$, and $\mathrm{Dt}=0.34 \mathrm{eV}$.

The approach taken here for the calculation of intrinsic linewidths is largely based on that outlined by Okada et al. ${ }^{10}$ A state $|e\rangle$ of the core-hole excited configuration $3 \mathrm{p}^{5} 3 \mathrm{~d}^{\mathrm{N}+1}$ can undergo Auger decay, leaving behind an ion core with electronic state $|f\rangle$ while emitting a free electron $|\varepsilon l\rangle$ with kinetic energy $\varepsilon$ and orbital angular momentum $l$ relative to the nucleus. The combined final state is denoted $|f \varepsilon l\rangle$. The intrinsic linewidth of state $|e\rangle$ is then given by $\Gamma_{e}=\pi \sum_{f \varepsilon l}\left|V_{e f}(\varepsilon l)\right|^{2}$ where the summation extends over all combinations of $|f\rangle$ and $|\varepsilon l\rangle$ that satisfy energy conservation, and where $V_{e f}(\varepsilon l)=\left\langle e\left|\frac{1}{r}\right| f \varepsilon l\right\rangle$ in which $\frac{1}{r}$ denotes the electronelectron coulombic operator acting between two configurations. Strictly speaking, $V_{e f}(\varepsilon l)$ is a non-constant function of $\varepsilon$, albeit a smoothly varying one. ${ }^{1}$ To simplify the computational procedure, a nominal representative value of $\varepsilon$, determined from published Auger kinetic energies or from $3 p$ binding energies if the former is not available, is used to calculate all $V_{e f}(\varepsilon l)$. With this simplification, the approximate intrinsic linewidth is given by $\Gamma_{e}=$ $\pi \sum_{f l}\left|V_{e f}(\bar{\varepsilon} l)\right|^{2}$ where $\bar{\varepsilon}$ is the nominal kinetic energy.

Under the constraints of selection rules, only $l=1,3$ or 5 are relevant for the decay of a $3 \mathrm{p}^{5} 3 \mathrm{~d}^{\mathrm{N}+1}$ state. In the formalism of atomic multiplet theory, the matrix elements $V_{e f}(\bar{\varepsilon} l)$ are determined by radial configuration interaction integrals $R^{k}(d d, p \bar{\varepsilon} p)$ and $R^{k}(d d, p \bar{\varepsilon} f)$ where $k=1$ or 3 and $R^{3}(d d, p \bar{\varepsilon} h) .{ }^{1}$ These quantities are calculated by CTM4XAS together with the other radial integrals such as the Slater-Condon parameters, and downscaled as appropriate.

With the calculated linewidths, the intrinsic line shape for a transition to state $|e\rangle$ is given by $f(E)=\left(1-\frac{1}{q^{2}}\right) \frac{\Gamma}{\pi\left(E^{2}+\Gamma^{2}\right)}+\frac{2}{q} \frac{E}{\pi\left(E^{2}+\Gamma^{2}\right)}$, where $q$ is an asymmetry parameter reflecting the competition between $3 \mathrm{~d}$ direct photoionization and a $3 \mathrm{p}-3 \mathrm{~d}$ transition. ${ }^{11}$ As the asymmetry parameter $q$ becomes larger, there is less competition from direct photoionization and the line shape becomes more symmetric. This intrinsic line shape is convoluted with a Gaussian function of $0.2 \mathrm{eV}$ sigma to give the simulated line shape. 


\section{Analysis of $\mathbf{M}_{2,3}$-edge spectral features}

It is instructive to compare $\mathrm{Co}^{\mathrm{III}}(\mathrm{acac})_{3}$ and $\mathrm{Fe}^{\mathrm{II}} \mathrm{Tren}(\mathrm{Py})_{3}{ }^{2+}$, which both have low-spin $3 d^{6}$ configurations. Both complexes give spectra with three main peaks, reflecting their analogous electronic structure. The $\mathrm{Co}^{\text {III }}(\mathrm{acac})_{3}$ spectrum is blue-shifted compared to that of $\mathrm{Fe}^{\mathrm{II}} \mathrm{Tren}(\mathrm{Py})_{3}{ }^{2+}$, as expected from the greater binding energy of the Co $3 p$ shallow-core orbitals compared to $\mathrm{Fe} 3 p$. The three-peak structure can be understood by constructing an excited-state analogue of a Tanabe-Sugano diagram, as shown in Figure $\mathrm{S} 2 \mathrm{for} \mathrm{Fe}^{\mathrm{II}}$ (the $\mathrm{Co}^{\mathrm{III}}$ case is analagous). Spin-orbit coupling is ignored for the moment, but will be reintroduced below. In the weak-field limit $(10 \mathrm{Dq}=0 \mathrm{eV})$, the lowest $\mathrm{d}^{6}$ singlet state ${ }^{1} \mathrm{I}_{\mathrm{g}}$ can access two ${ }^{1} \mathrm{H}_{\mathrm{u}}$ states and one ${ }^{1} \mathrm{I}_{\mathrm{u}}$ state in the $3 \mathrm{p}^{5} 3 \mathrm{~d}^{7}$ manifold by electric dipole transitions. The transitions to the two ${ }^{1} \mathrm{H}_{\mathrm{u}}$ states interfere such that the strength of the higher-energy transition is enhanced, analogous to the strong Soret and weak Q bands in the UV/Vis spectra of porphyrins. ${ }^{12}$ Note that at zero field the ground state is actually a quintet; we show the singlet state here as a means to understand the spectra at intermediate and high fields.

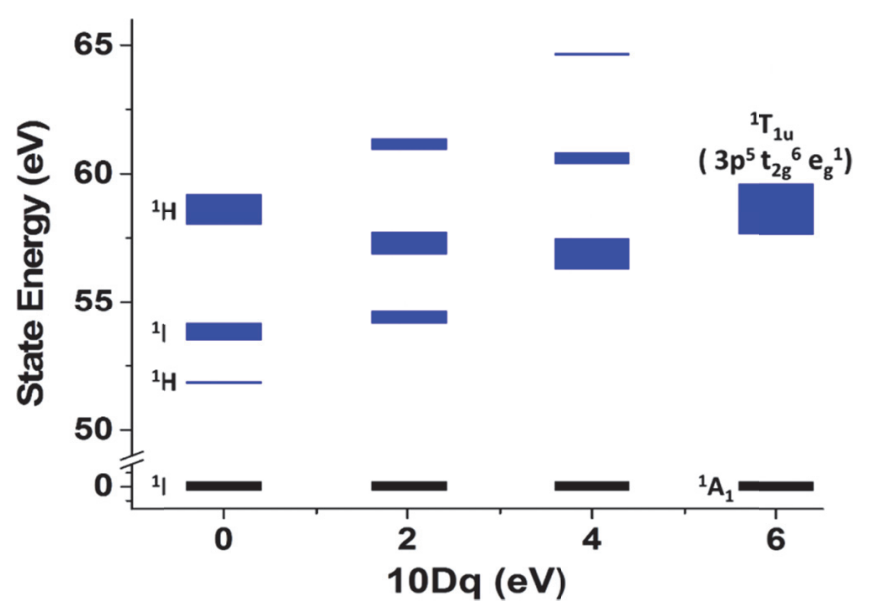

Figure S2: Effect of the ligand field strength on singlet excited-state terms of $\mathrm{Fe}^{\mathrm{II}} \mathrm{Tren}(\mathrm{Py})_{3}{ }^{2+}$. In the limit of zero ligand field, transitions are allowed from the lowest singlet state ${ }^{1} \mathrm{I}$ to ${ }^{1} \mathrm{H}$ and ${ }^{1} \mathrm{I}$ core-hole excited states. As the ligand field strength increases, these states mix and shift in energy. In the strong-field limit, a single transition from the ${ }^{1} \mathrm{~A}_{1}$ ground state to the ${ }^{1} \mathrm{~T}_{1 \mathrm{u}}$ core-hole state is allowed. The size of the blue box is proportional to the oscillator strength of the transition. A simulated spectrum of the $10 \mathrm{Dq}=2 \mathrm{eV}$ case is shown in Figure $\mathrm{S} 3$

When an octahedral ligand field is applied to the $\mathrm{Fe}^{\mathrm{II}}$ center, its ground state eventually takes on the symmetry of ${ }^{1} \mathrm{~A}_{\mathrm{g}}$ as the ligand field strength overcomes $3 d-3 d$ electron repulsion. At this point, the ligand field is sufficiently strong to induce splitting and mixing of the free ion states. Under the reduced symmetry, the number of allowed transitions increases, but the overall spectrum is dominated by three major transitions of comparable intensities. A simulated spectrum with $10 \mathrm{Dq}=2.0 \mathrm{eV}$ is shown in Figure S3A. For illustrative purposes, a narrow $0.2 \mathrm{eV}$ Lorentzian linewidth is used at this point.

As the strength of ligand field is further increased, intensity is redistributed to the lowest energy transition due to re-mixing of states in the $3 p^{5} 3 d^{7}$ manifold. In the limit of high ligand field strength $(10 \mathrm{Dq}=6 \mathrm{eV})$, a single transition to a ${ }^{1} \mathrm{~T}_{1 \mathrm{u}}$ term is predicted. This state is a linear

combination of the ${ }^{1} I_{u}$ and ${ }^{1} \mathrm{H}_{u}$ terms, along with contributions from higher-energy singlet terms. 
Spin-orbit coupling of the $3 p$ core-hole in the final state mixes these transitions and adds several new small features while preserving the general three-peak shape (Figure S3B). Finally, the peaks are broadened in Figure S3C with a Gaussian function, a Fano line shape, and a Lorentzian linewidth as described above.

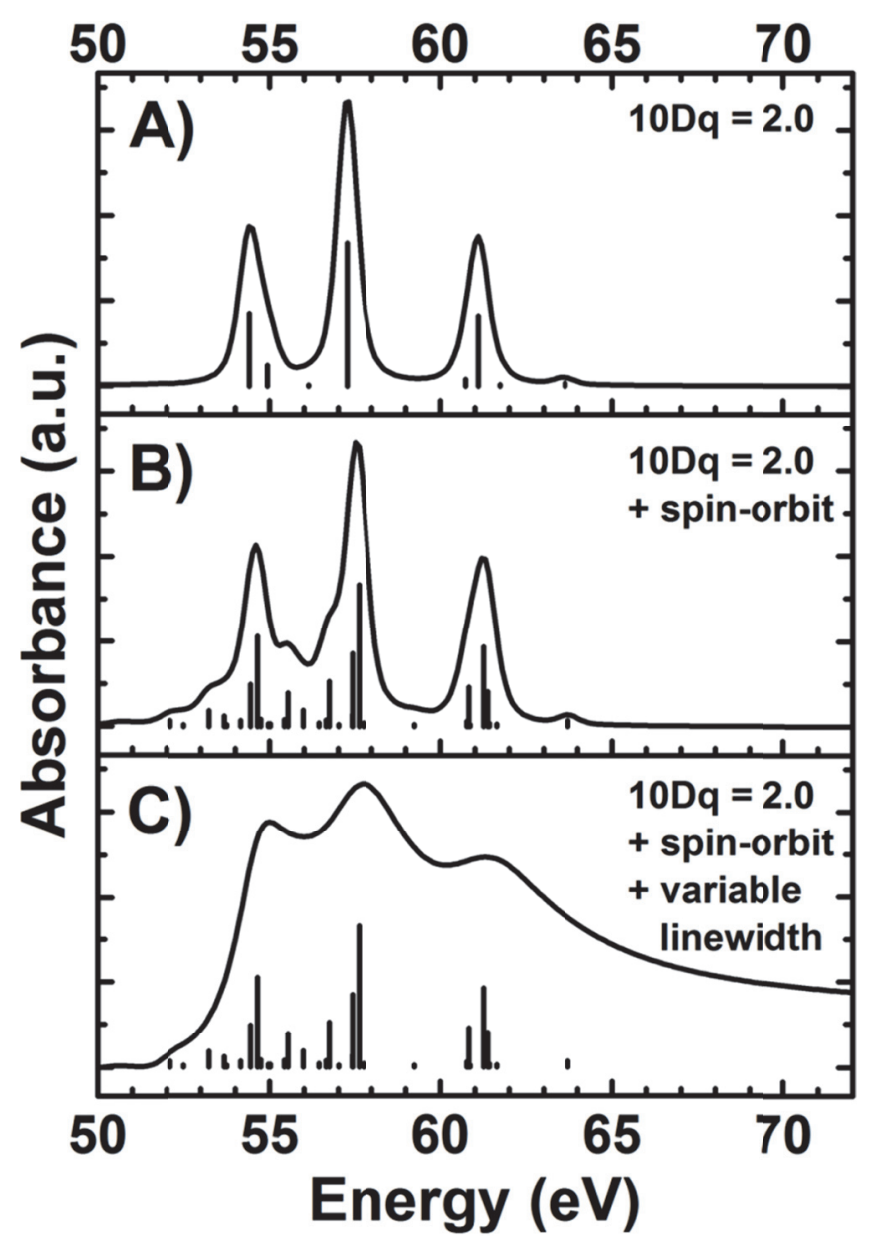

Figure S3: Ligand field multiplet spectra of a $d^{6} \mathrm{Fe}^{\mathrm{II}}$ ion in an octahedral crystal field with $10 \mathrm{Dq}=2 \mathrm{eV}$. (A) Simulation with no spin-orbit coupling and $\Gamma=0.2 \mathrm{eV}$ Lorentzian linewidths shows three main peaks. (B) Inclusion of spin-orbit coupling adds additional transitions but preserves the overall shape. (C) Final simulated spectrum with $0.2 \mathrm{eV}$ Gaussian broadening, Fano line shapes, and a term-dependent Lorentzian linewidth.

The simulated spectra of all five molecules studied, along with peak assignments, are shown below (including $\mathrm{Fe}^{\mathrm{II}} \mathrm{Tren}(\mathrm{Py})_{3}{ }^{2+}$ for easy comparison between the spectra). For clarity in the assignments, spin-orbit coupling is not included in the calculation. The simulated spectra are therefore slightly different from those presented in the main text. All core-hole excited states have ungerade symmetry, so the ' $u$ ' symmetry designation is not shown. 

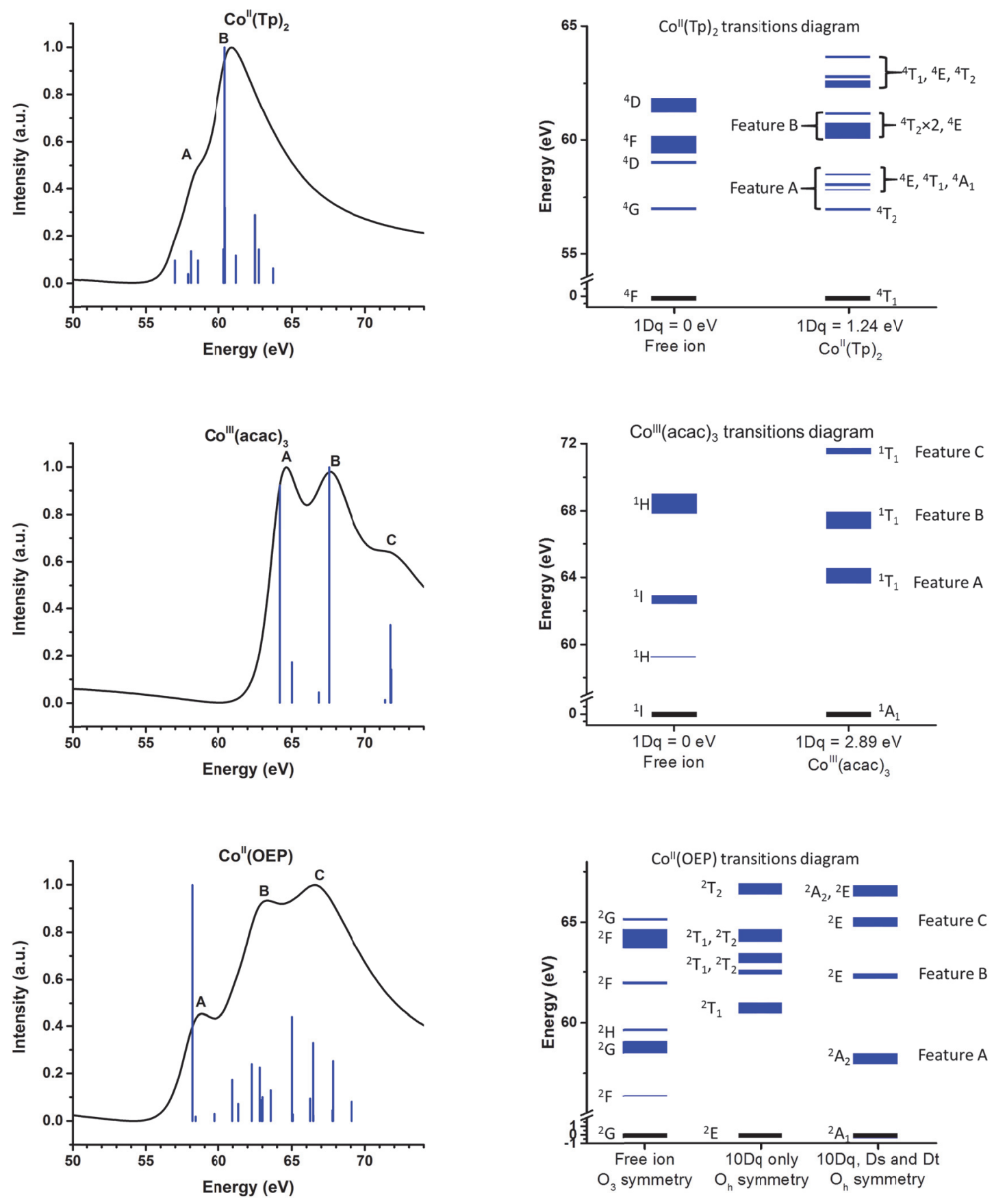

Figure S4: Peak assignments for Co complexes. Note that the sticks are at slightly lower energy than the peaks in the broadened spectrum due to the Fano lineshape. 

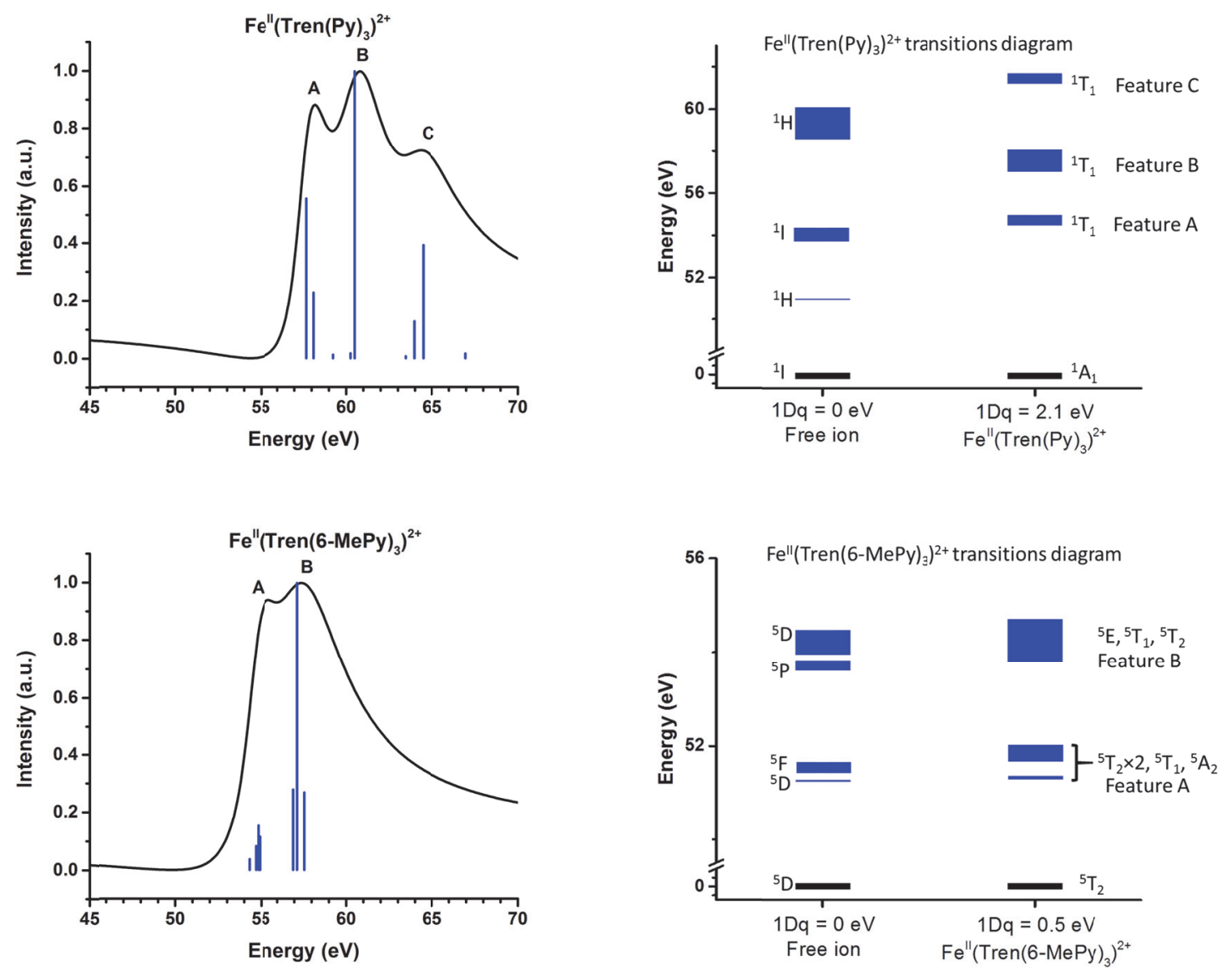

Figure S5: Peak assignments for Fe complexes

These transitions can also be accessed using $1 \mathrm{~s} 3 \mathrm{p}$ RIXS spectra. ${ }^{13}$ Projection of a 2D-RIXS spectrum onto the energy transfer axis by integrating along the incident energy axis results in a spectrum similar to a $3 p-3 d$ absorption spectrum (analogous to the relationship between $1 \mathrm{~s} 2 \mathrm{p}$ RIXS and $\mathrm{L}_{2,3}$-edge absorption) ${ }^{14}$. Because the final states reached by an $1 \mathrm{~s} 3 \mathrm{p}$ RIXS process also contain $3 p$-core-holes, the peaks in the spectrum are broadened to a similar extent as are those in an ordinary $\mathrm{M}_{2,3}$-edge spectrum. On the other hand, due to the coherent involvement of an intermediate state, this spectrum may include transitions that are forbidden in a direct $3 p-3 d$ absorption process and the intensities of peaks may also be different from what one would observe in an ordinary $3 p-3 d$ absorption spectrum. As in the $1 s 2 p$ RIXS case, the full $2 D$ 1s $3 p$ RIXS map may help to separate overlapping absorption features. In addition, use of hard x-rays would allow for much thicker samples to be measured, and in heterometallic samples would make it easier to pinpoint a single element.

\section{Post-edge intensity}


Although the general shape and peak spacings in the simulated spectra in Figure 3 of the main text match those in the experimental spectra, the experimental spectra show a broad post-edge absorption that is absent in the simulations. In some of our spectra, such as those for $\mathbf{C o}^{\text {III }}$ (acac) $)_{3}$ and $\mathbf{C o}^{\text {II }}(\mathbf{O E P})$, the experimental absorbance continues to rise with increasing energy, at least up to the $72 \mathrm{eV}$ limit of our instrument. This behavior is likely due to additional absorption channels that are not included in the simulation, primarily photoionization of $3 p$ shallow-core electrons with a small contribution from $3 p \rightarrow 4 s$ absorption. At the $\mathrm{K}$ and $\mathrm{L}$ edges, the photoionization channel is often modeled as an arctangent function, with an amplitude chosen empirically to fit the pre-edge and post-edge plateaus. ${ }^{15}$ Addition of such a function to the simulations recovers the high post-edge absorption, as shown for $\mathbf{C o}^{\text {II }}(\mathbf{T} \mathbf{p})_{2}$ in Figure S6. In this case, the baseline correction also results in a better match of the intensity ratio of the shoulder and main peak (compared to the simulation in Figure 4B). However, this baseline adds three additional simulation parameters (center position, step width, and amplitude of the arctangent function) and increases the risk of overfitting. Because the post-edge height is dependent on contributions from both $3 p$ photoionization processes and the Fano asymmetry, it is difficult to separate out the relative contributions of these two factors.

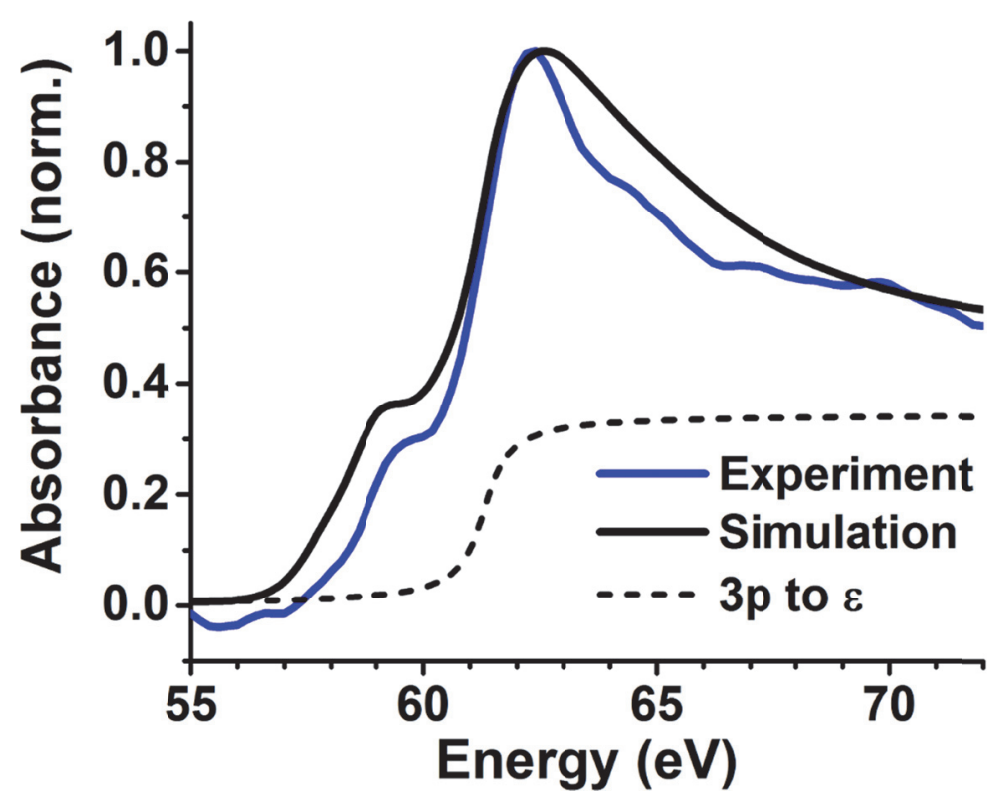

Figure S6: Experimental and simulated spectra of $\mathrm{Co}^{\mathrm{II}}(\mathrm{Tp})_{2}$ including an arctangent baseline to account for $3 p$ photoionization. Addition of this $3 p \rightarrow \varepsilon$ channel improves the peak height ratio and high-energy plateau compared to Figure $3 \mathrm{C}$, at the expense of additional adjustable parameters. 


\section{Sample preparation}

Bis[hydrotris(pyrazolyl)borato]cobalt(II) $\left(\mathrm{Co}^{\mathrm{II}}(\mathrm{Tp})_{2}\right),{ }^{16}[\operatorname{tris}(2-(($ pyridin-2ylmethylene)amino)ethyl)amine]iron(II) hexafluorophosphate $\left(\mathrm{Fe}^{\mathrm{II}} \mathrm{Tren}(\mathrm{Py})_{3}\left(\mathrm{PF}_{6}\right)_{2}\right)$ and $[\operatorname{tris}(2-$ ((6-methylpyridin-2-ylmethylene)amino)ethyl)amine]iron(II) hexafluorophosphate ( $\mathrm{Fe}^{\mathrm{II}} \operatorname{Tren}(6-$ $\left.\mathrm{MePy})_{3}\left(\mathrm{PF}_{6}\right)_{2}\right)^{17}$ were synthesized according to published procedures. Tris(acetylacetonato)cobalt(II) $\left(\mathrm{Co}^{\mathrm{III}}(\mathrm{acac})_{3}\right)$ and octaethylporphyrinato)cobalt(II) (Co $\left.\mathrm{CI} O \mathrm{OEP}\right)$ were purchased from Sigma Aldrich. Samples were deposited as $\sim 100 \mathrm{~nm}$ thick films on $100 \mathrm{~nm}$ silicon nitride membranes by either thermal evaporation (Co complexes) or spin-casting from DMF solutions (Fe complexes).

\section{Data Collection and Baseline Subtraction}

Absorption spectra are collected in transmission geometry by cycling between a samplecoated membrane and a bare silicon nitride membrane, both mounted on an in-vacuum motorized stage. A typical spectrum takes five minutes to collect, alternating between ten 0.5 second exposures of the sample and ten exposures of the blank. The spectra presented here are the average of at least three spectra taken on three different days. No sample damage due to the XUV beam was seen even after illumination for one hour on one spot, as tested by comparisons with unilluminated regions of the sample. The spectra are collected in $0.2 \mathrm{eV}$ bins, followed by five-point adjacent-average smoothing. Note that the residual noise in the spectra is an instrumental artifact caused by nonlinearity in the CCD detector and spatial inhomogeneity in the XUV beam - further averaging does not reduce the small-scale fluctuations. We have recently analyzed these artifacts in detail. ${ }^{18}$ The spectrometer is calibrated daily using the Al L edge at $72.7 \mathrm{eV}$, its second-order diffraction feature at $36.35 \mathrm{eV}$, and the $\alpha-\mathrm{Fe}_{2} \mathrm{O}_{3}$ peak at $57.5 \mathrm{eV}^{4}$. This calibration is verified periodically using the sharp atomic resonances of Xe at 65.11 and 67.04 $\mathrm{eV}^{19}$ The noise in the spectra suggests that the reported peak positions should be considered accurate to $0.2 \mathrm{eV}$. 


\section{Raw spectra and baselines}

The figures below show a single spectrum for each molecule referenced to a bare $\mathrm{Si}_{3} \mathrm{~N}_{4}$ substrate before baseline subtraction, along with the power law baseline. After subtraction, the spectrum is normalized, and several such normalized spectra are averaged for each of the final spectra shown in Figure 3 of the main text. The baseline is fit for at least $5 \mathrm{eV}$ before the absorption edge. Below $50 \mathrm{eV}$, the photon flux is low and factors such as detector nonlinearity and stray light cause the apparent absorption to drop below the baseline. Also shown below are the averaged spectra with error bars for each point ( \pm standard deviation). Note that for the Co samples, the harmonic conditions were optimized for maximum flux and stability in the 60-72 $\mathrm{eV}$ range, so $\mathrm{Co}^{\mathrm{II}}(\mathrm{acac})_{3}$ and $\mathrm{Co}^{\mathrm{III}}(\mathrm{OEP})$ exhibit significant systematic noise below the absorption edge due to low XUV flux. This manifests in artifacts every $3.1 \mathrm{eV}$ in the low-flux regions, as discussed above in Section 6 .
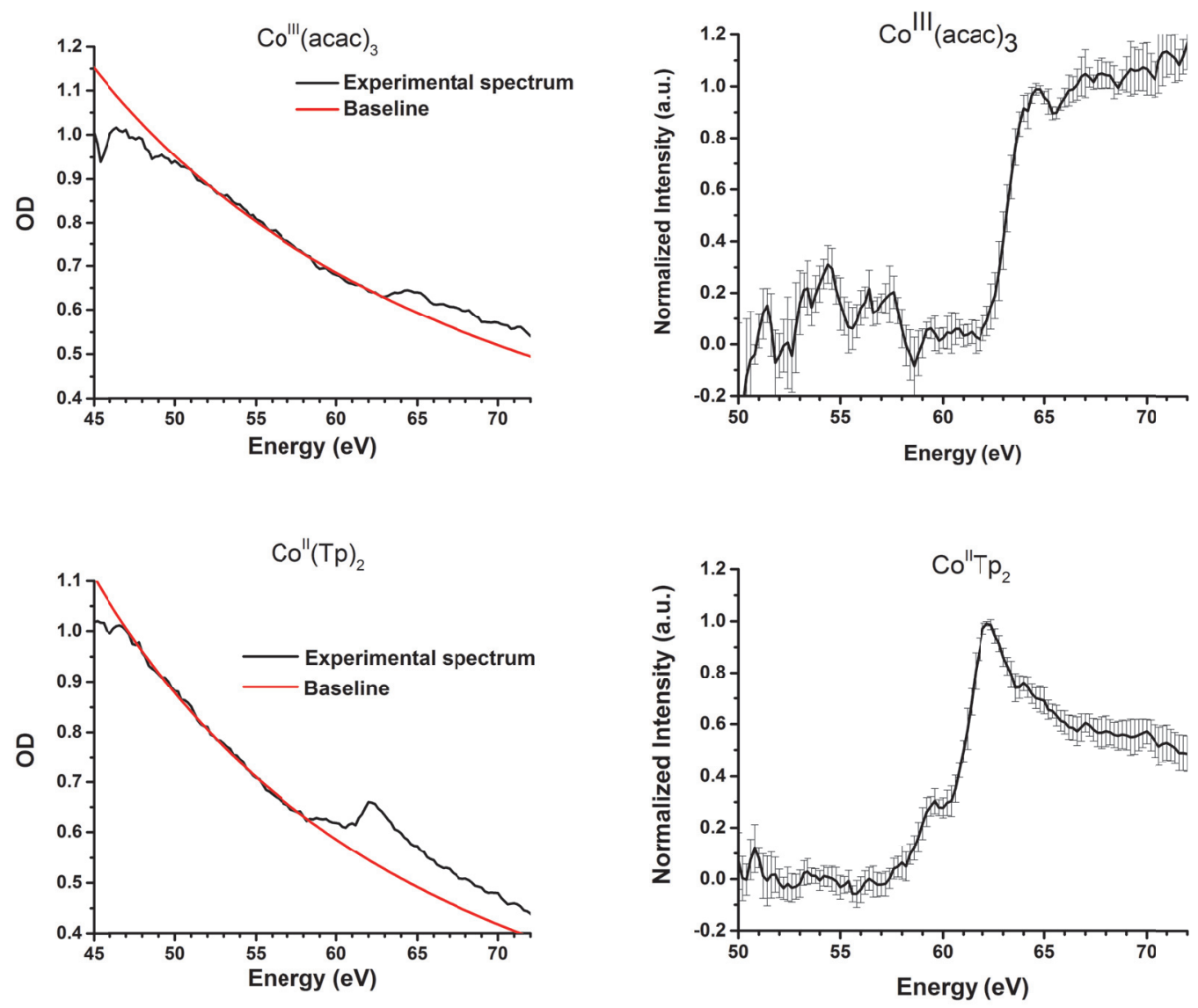

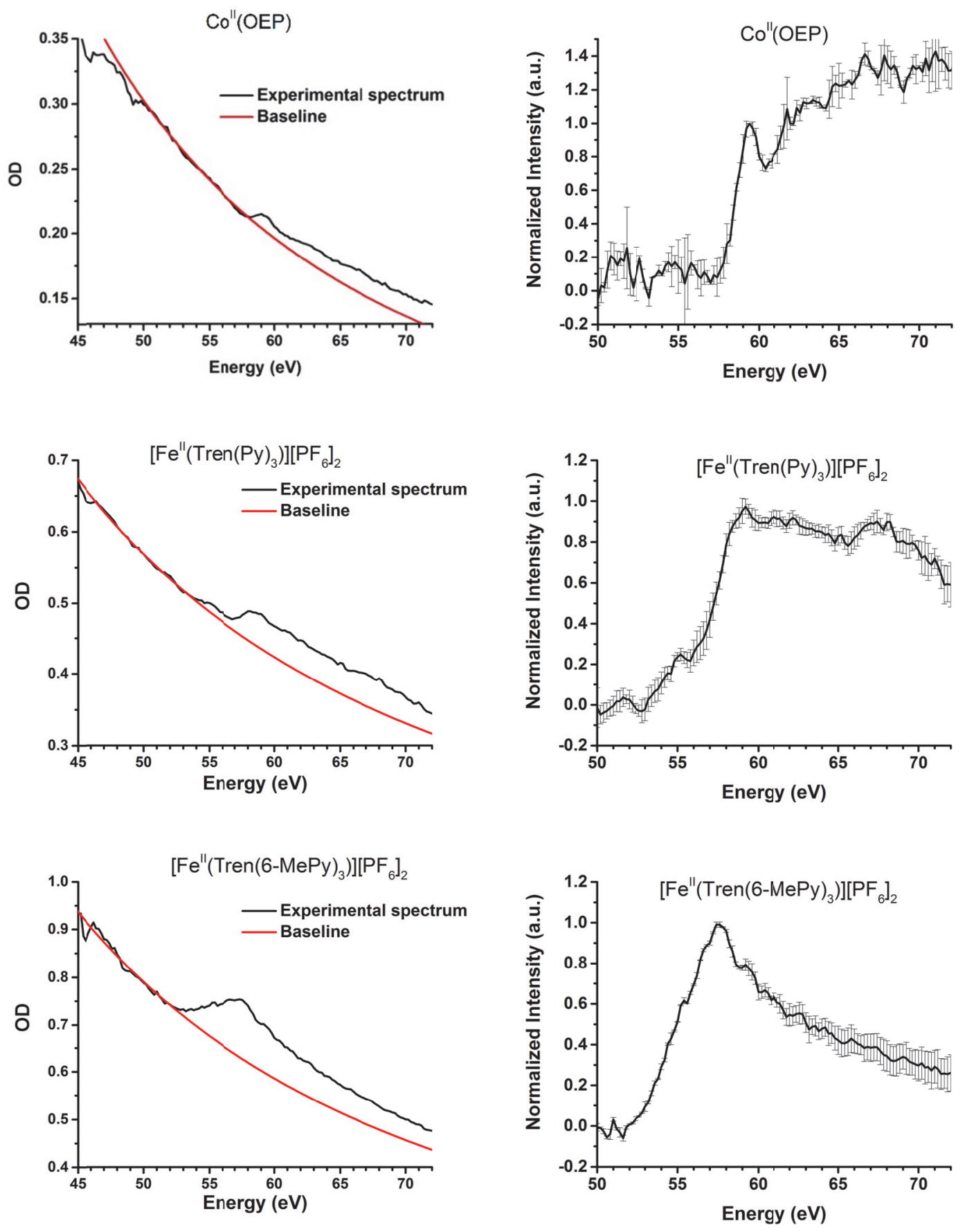

Figure S7: Spectra of coordination complexes before baseline subtraction 


\section{References for Supporting Information}

(1) Cowan, R. . Theory of Atomic Structure and Spectra; University of California Press: Berkeley, CA, 1981.

(2) Thole, B. T.; Van Der Laan, G.; Butler, P. H. Spin-Mixed Ground State of Fe Phthalocyanine and the Temperature-Dependent Branching Ratio in X-Ray Absorption Spectroscopy. Chem. Phys. Lett. 1988, 149, 295-299.

(3) Berlasso, R.; Dallera, C.; Borgatti, F.; Vozzi, C.; Sansone, G.; Stagira, S.; Nisoli, M.; Ghiringhelli, G.; Villoresi, P.; Poletto, L.; et al. High-Order Laser Harmonics and Synchrotron Study of Transition Metals $\mathrm{M}_{2,3}$ Edges. Phys. Rev. B 2006, 73, 115101.

(4) Vura-Weis, J.; Jiang, C.-M.; Liu, C.; Gao, H.; Lucas, J. M.; de Groot, F. M. F.; Yang, P.; Alivisatos, A. P.; Leone, S. R. Femtosecond $\mathrm{M}_{2,3}$-Edge Spectroscopy of Transition-Metal Oxides: Photoinduced Oxidation State Change in $\alpha-\mathrm{Fe}_{2} \mathrm{O}_{3}$. J. Phys. Chem. Lett. 2013, 4, 3667-3671.

(5) Butler, P. H. Point Group Symmetry Applications, Methods and Tables; Plenum Press: New York, 1981.

(6) Jesson, J. P. Optical and Paramagnetic Resonance Spectra of Some Trigonal Co(II) Chelates. J. Chem. Phys. 1966, 45, 1049.

(7) Ballhausen, C. J. Introduction to Ligand Field Theory; McGraw Hill, 1962.

(8) Huse, N.; Kim, T. K.; Jamula, L.; McCusker, J. K.; de Groot, F. M. F.; Schoenlein, R. W. Photo-Induced Spin-State Conversion in Solvated Transition Metal Complexes Probed via Time-Resolved Soft X-Ray Spectroscopy. J. Am. Chem. Soc. 2010, 132, 6809-6816.

(9) Liao, M.-S.; Scheiner, S. Electronic Structure and Bonding in Metal Porphyrins, metal=Fe, Co, Ni, Cu, Zn. J. Chem. Phys. 2002, 117, 205.

(10) Okada, K.; Kotani, A.; Ogasawara, H.; Seino, Y.; Thole, B. T. Auger Decay of QuasiParticle States - Calculation of the Ni 3p Photoemission Spectrum in $\mathrm{NiCl}_{2}$. Phys. Rev. B 1993, 47, 6203-6206.

(11) Fano, U. Effects of Configuration Interaction on Intensities and Phase Shifts. Phys. Rev. 1961, 1, 1866-1878.

(12) Gouterman, M. Spectra of Porphyrins. J. Mol. Spectrosc. 1961, 6, 138-163.

(13) Glatzel, P.; Bergmann, U.; de Groot, F. M. F.; Weckhuysen, B. M.; Cramer, S. P. A Study of Transition Metal K Absorption Pre-Edges by Resonant Inelastic X-Ray Scattering (RIXS). Phys. Scr. 2005, T115, 1032.

(14) Glatzel, P.; Bergmann, U.; Gu, W.; Wang, H.; Stepanov, S.; Mandimutsira, B. S.; Riordan, C. G.; Horwitz, C. P.; Collins, T.; Cramer, S. P. Electronic Structure of Ni Complexes by X-Ray Resonance Raman Spectroscopy (Resonant Inelastic X-Ray Scattering). J. Am. Chem. Soc. 2002, 124, 9668-9669. 
(15) Hocking, R. K.; Wasinger, E. C.; de Groot, F. M. F.; Hodgson, K. O.; Hedman, B.; Solomon, E. I. Fe L-Edge XAS Studies of $\mathrm{K}_{4}\left[\mathrm{Fe}(\mathrm{CN})_{6}\right]$ and $\mathrm{K}_{3}\left[\mathrm{Fe}(\mathrm{CN})_{6}\right]$ : A Direct Probe of Back-Bonding. J. Am. Chem. Soc. 2006, 128, 10442-10451.

(16) Trofimenko, S.; Long, J. R.; Nappier, T.; Shore, S. G. Poly(1-Pyrazolyl)borates, Their Transition-Metal Complexes, and Pyrazaboles. In Inorganic Syntheses; John Wiley \& Sons, Inc., 2007; pp 99-109.

(17) Hoselton, M. A.; Wilson, L. J.; Drago, R. S. Substituent Effects on the Spin Equilibrium Observed with Hexadentate Ligands on iron(II). J. Am. Chem. Soc. 1975, 97, 1722-1729.

(18) Lin, M.-F.; Verkamp, M. A.; Ryland, E. S.; Zhang, K.; Vura-Weis, J. Impact of Spatial Chirp on High Harmonic Extreme Ultraviolet Absorption Spectroscopy of Thin Films. $J$ Opt Sci Am B; In Press 2016.

(19) Ederer, D. L.; Manalis, M. Photoabsorption of the 4d Electrons in Xenon. J. Opt. Soc. Am. 1975, 65, 634.

\section{Full references for citations 10, 11, 13, 17, 24, and 34 in the main text}

(10) Turgut, E.; La-o-Vorakiat, C.; Shaw, J. M.; Grychtol, P.; Nembach, H. T.; Rudolf, D.; Adam, R.; Aeschlimann, M.; Schneider, C. M.; Silva, T. J.; Murnane, M. M.; Kapteyn, H. C.; Mathias, S. Controlling the Competition between Optically Induced Ultrafast SpinFlip Scattering and Spin Transport in Magnetic Multilayers. Phys. Rev. Lett. 2013, 110, 197201.

(11) La-O-Vorakiat, C.; Siemens, M.; Murnane, M. M.; Kapteyn, H. C.; Mathias, S.; Aeschlimann, M.; Grychtol, P.; Adam, R.; Schneider, C. M.; Shaw, J. M.; Nembach, H.; Silva, T. J. Ultrafast Demagnetization Dynamics at the M Edges of Magnetic Elements Observed Using a Tabletop High-Harmonic Soft X-Ray Source. Phys. Rev. Lett. 2009, 103,257402 .

(13) Goulielmakis, E.; Loh, Z. H.; Wirth, A.; Santra, R.; Rohringer, N.; Yakovlev, V. S.; Zherebtsov, S.; Pfeifer, T.; Azzeer, A. M.; Kling, M. F.; Leone, S.R.; Krausz, F. RealTime Observation of Valence Electron Motion. Nature 2010, 466, 739-743.

(17) Popmintchev, T.; Chen, M. C.; Popmintchev, D.; Arpin, P.; Brown, S.; Alisauskas, S.; Andriukaitis, G.; Balciunas, T.; Mucke, O. D.; Pugzlys, A.; Baltuska, A.; Shim, B.; Schrauth, S. E.; Gaeta, A.; Hernandez-Garcia, C. ; Plaja,; Becker, A.; Jaron-Becker, A.; Murnane, M. M.; Kapteyn, H. C. Bright Coherent Ultrahigh Harmonics in the keV X-Ray Regime from Mid-Infrared Femtosecond Lasers. Science 2012, 336, 1287-1291.

(24) Berlasso, R.; Dallera, C.; Borgatti, F.; Vozzi, C.; Sansone, G.; Stagira, S.; Nisoli, M.; Ghiringhelli, G.; Villoresi, P.; Poletto, L.; Pascolini, M.; Nannarone, S.; De Silvestri, S.; Braicovich, L. High-Order Laser Harmonics and Synchrotron Study of Transition Metals $\mathrm{M}_{2,3}$ Edges. Phys. Rev. B 2006, 73, 115101. 
(34) Guda, S. A.; Guda, A. A.; Soldatov, M. A.; Lomachenko, K. A.; Bugaev, A. L.; Lamberti, C.; Gawelda, W.; Bressler, C.; Smolentsev, G.; Soldatov, A. V.; Joly, Y. Optimized Finite Difference Method for the Full-Potential XANES Simulations: Application to Molecular Adsorption Geometries in MOFs and Metal-Ligand Intersystem Crossing Transients. $J$. Chem. Theory Comput. 2015, 11, 4512-4521. 